\title{
Recertification of the SRM 706a, a Polystyrene
}

\author{
C. M. Guttman \\ W. R. Blair \\ J. R. Maurey \\ Polymers Division
}

U.S. DEPARTMENT OF COMMERCE

Technology Administration

National Institute of Standards and Technology Gaithersburg, MD 20899-0001

QC 


\section{NISTIR 6091}

\section{Recertification of the SRM 706a, a Polystyrene}

\author{
C. M. Guttman \\ W. R. Blair \\ J. R. Maurey \\ Polymers Division
}

U.S. DEPARTMENT OF COMMERCE

Technology Administration

National Institute of Standards and Technology

Gaithersburg, MD 20899-0001

September 1998

Final Report prepared for

Standard Reference Materials Program

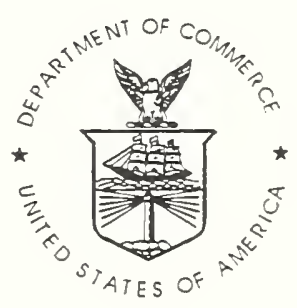

U.S. DEPARTMENT OF COMMERCE

William M. Daley, Secretary

TECHNOLOGY ADMINISTRATION

Gary R. Bachula, Acting Under Secretary for Technology

NATIONAL INSTITUTE OF STANDARDS

AND TECHNOLOGY

Raymond G. Kammer, Director 
Recertification of SRM 706a, a Polystyrene

by C. M. Guttman, W.R. Blair and J. R. Maurey

Polymers Division

N.I.S.T.

Gaithersburg, MD 20899

Certain commercial materials and equipment are identified in this paper in order to specify adequately the experimental procedure. In no case does such identification imply recommendation or endorsement by the National Institute of Standards and Technology, nor does it imply necessarily the best available for the purpose.

According to ISO 31-8, the term "Molecular Weight" has been replaced by "Relative Molecular Mass," symbol $M_{r}$. Thus, if this nomenclature and notation were followed in this publication, one should write $M_{\mathrm{r}, \mathrm{w}}$ instead of the historically conventional $\mathrm{M}_{\mathrm{w}}$ for the weight average molecular weight with similar changes for $M_{n}, M_{z}$, and $M_{v} . M_{w}$ would be called the "Mass Average Relative Molecular Mass." The conventional notation, rather than the ISO notation has been used in this publication.

A final report prepared for the sponsors, Standard Reference Materials Program 


\section{ABSTRACT}

The recertification of the polystyrene standard reference material, SRM 706a, is described. The $M_{w}$ of SRM 706a by light scattering was determined to be $2.85 \times 10^{5}$ $\mathrm{g} / \mathrm{mol}$ with a sample standard deviation of $0.019 \times 10^{5} \mathrm{~g} / \mathrm{mol}$. A combined expanded uncertainty of $0.23 \times 10^{5} \mathrm{~g} / \mathrm{mol}$ is estimated for this determination. The $M_{w}$ obtained for this recertification agrees with the $M_{w}$ of $2.87 \times 10^{5} \mathrm{~g} / \mathrm{mol}$ obtained from recent light scattering measurements on SRM 706. The $M_{w}$ obtained for SRM 706a is also in excellent agreement with the $M_{w}$ obtained by ultracentrifugation in 1967 of $2.88 \times 10^{5}$ $\mathrm{g} / \mathrm{mol}$ on SRM 706. Bottle-to-bottle variability was found to be below detectable levels for SRM 706a in a size exclusion chromatography study. 


\subsection{Introduction}

This report describes the recertification of the $M_{w}$ of a polystyrene, SRM 706a. This material was originally certified in 1967[1]. Some original material was stored in a climate-controlled warehouse by the Standard Reference Materials Program (SRMP) for the $\mathbf{3 0}$ intervening years. After packaging of this material for future distribution, the $M_{w}$ was determined by light scattering. The bottle-to-bottle homogeneity of this Standard Reference Material (SRM) was studied by size exclusion chromatography (SEC).

2.0 Preparation, Bottling, and Sampling of SRM 706a

\subsection{Preparation}

The $M_{w}$ standard, SRM 706a, was prepared for certification at NIST. The preparation, purity and original homogeneity determinations are described in a report [1]. The as-received material was in the form of pellets.

\subsubsection{Bottling and Sampling of SRM 706a}

In the following, the containers holding SRM 706a and SRM 706 will be referred to as vials. A total of 650 samples of SRM 706a, about $1.0 \mathrm{~g}$ each, was bottled in amber vials. The entire set of samples was divided into 12 subsets. One vial was randomly selected from each subset of SRM 706a for homogeneity testing. Four vials of the original bottling, made 30 years ago, of SRM 706 were obtained from SRMP. These samples of SRM 706 were used in the subsequent studies to determine whether the rebottled material was in any way different from the originally bottled material.

\subsection{Homogeneity Testing on SRM 706a}

Homogeneity testing was accomplished using SEC. A Waters 150-C AL/GPC Liquid Chromatograph (Waters Corp., Milford, MA) with a differential refractive index (DRI) detector and three PL gel $300 \mathrm{~mm} \times 7.5 \mathrm{~mm}$ ID $10 \mu \mathrm{m}$ MIXED-B SEC (Polymer Labs, Amherst, MA) columns were used in this study. The chromatography was run at $1.0 \mathrm{~mL} / \mathrm{min}$ solvent flow rate. The injector and column compartment of the Waters 150 $\mathrm{C}$ were controlled at $30^{\circ} \mathrm{C}$ for all measurements. Mallinkrodt Tetrahydrofuran (Mallinkrodt Specialty Chemicals, Paris, KY) with added antioxidant, 2,6-di-tert-butyl-4methyl phenol (commonly known as butylated hydroxytoluene or BHT), was used as the solvent. Additional $\mathrm{BHT}$ at $0.3 \mathrm{~g} / \mathrm{L}$ was added to the solvent used in preparing solutions as a SEC pump marker.

Single pellets from each vial were used to prepare solutions. Two solutions were made from each vial. The polystyrene samples were dissolved in the solvent at 
concentration of approximately $1.0 \mathrm{~g} / \mathrm{L}$. Each bottle was shaken at frequent intervals to aid the dissolution. Each solution was filtered through a $0.45 \mu \mathrm{m}$ Acrodisc CR PTFE (Gelman Sciences, Ann Arbor, MI) disposable syringe filter. The order of preparing the solutions and running the chromatograms was randomized using a method described in section 1-4 of Natrella [2]. SEC was performed on these solutions using two injections from each solution.

After baseline subtraction, the SEC chromatograms were normalized to unit peak height and compared initially by overlaying to decide if there were visible differences outside the noise. The chromatograms from different solutions all superimpose on each other. This preliminary comparison showed that polymer samples taken from all the vials produced identical chromatograms. In section 3.2, statistical analysis on the chromatography confirms these visual observations

\subsection{Statistical Method to Compare Chromatograms}

\subsubsection{Match Factor}

In previous SRM SEC studies the match factor was used to compare one chromatogram with all the others. In this study, the match factor for chromatogram I is defined as the correlation coefficient between chromatogram I and the average chromatogram of the entire testing series. The match factor is defined by Huber [3] as

Match Factor $\left.=10^{3}\left\{\sum x^{*} y-\left(\sum x^{*} \sum y\right) / p\right\}^{2} \pi\left\{\sum x^{2}-\sum x^{*} \sum x / p\right\}\left\{\sum y^{2}-\sum y^{*} \sum y / p\right\}\right]$.

The value of $x$ is the measured signal in the ith chromatogram and $y$ is the measured signal from the average chromatogram at the same elution time; $p$ is the number of data points in the chromatogram. The sums are taken over all data points.

At the extremes, a match factor of zero indicates no match and 1000 indicates an identical chromatogram. Generally, values above 990 indicate that the chromatograms are similar. Values between 900 and 990 indicate some similarity between chromatograms, but the result should be interpreted with care. All values below 900 are interpreted as an indication of different chromatograms [3,4].

An ANOVA study using OMNITAB [5] and DATAPLOT [6] made on the match factors obtained from the chromatograms indicated that the match factors of chromatograms from vials of SRM 706 and vials of SRM 706a were not different using a significance level with $\alpha=0.05$. The chromatograms were run in groups of seven solutions on different days. Using the match factor we found measurements on one particular day were different from the rest on a level of significance with $\alpha=0.05$. 
Using the Scheffe pairwise multiple comparison of the means of match factors from chromatograms from the same vial, no difference was observed between vials on a level of significance with $\alpha=0.05$ [2]. All chromatograms in this study had match factors against the mean chromatogram of greater than 999 . In the study of day to day variation, the mean of the single day outlier had a match factor of less than 0.2 different from the other day means.

As described above, BHT was added to the solutions of polymer and solvent, as a marker to indicate the reproducibility of the solvent volume delivered by the SEC pump for all the above measurements. The BHT peaks were widely separated from the peaks for the polystyrene, and thus could be separately analyzed. Interferences in the low molecular weight region made it difficult to obtain a full baseline-to-baseline chromatogram for the BHT. Thus, an analysis of the match factor method similar to that described for the hexadecane pump marker in the chromatographic study of SRM 1482a could not be done [7]. However, the peak position of the BHT was sufficiently separated from the other interferences and was sufficiently narrow that it could be used to estimate of the fidelity of the chromatography. A variation in the BHT peak position would indicate chromatographic system variations and not sample to sample variations. An ANOVA study using OMNITAB made on the match factors of the chromatograms of SRM 706 and SRM 706a versus the peak position of the BHT showed no correlation on a level of significance with $\alpha=0.05$.

From the above considerations, we conclude that the vials of SRM 706a are indistinguishable and there is no difference between SRM 706 and SRM 706a.

4.0 Determination of $M_{w}$ of SRM 706a by Light Scattering

\subsection{Light Scattering on the Polystyrene Standard}

\subsubsection{Solution and Solvent Preparation}

The polystyrene samples were weighed on an analytical balance with $0.01 \mathrm{mg}$ resolution in mass indication. Buoyancy corrections were applied. The SRM 706a pellets were weighed in a tared $3 \mathrm{~g}$ glass weighing boat. Pellets are of varying size weighing on average $100 \mathrm{mg}$ with a range from $60 \mathrm{mg}$ to $200 \mathrm{mg}$. At some of the lowest concentrations only one pellet was used. In the original report describing SRM 706 [1] no pellet-to-pellet variation in molecular mass was reported and no pellet to pellet variation was found from our SEC studies described in section 3.1.

Samples of SRM 706a were not subjected to special drying before or during weighing since PS pellets were found to pick up less than $0.0001 \mathrm{~g}$ in a $0.3 \mathrm{~g}$ sample upon sitting in a balance in humid air for $20 \mathrm{~min}$. 
The response of the balance was tested by weighing a $50 \mathrm{mg}$ standard balance weight. The balance appeared to arrive at equilibrium weight within $1 \mathrm{~min}$ after the 50 $\mathrm{mg}$ standard weight had been loaded onto the pan, and maintained the same indicated apparent weight within $\pm 0.00001 \mathrm{~g}$ random variation.

Analytical reagent grade toluene (Mallinckrodt Specialty Chemicals, Paris, KY) reagent, was used as the solvent without modification. The amount of solvent added was determined gravimetrically on a top loading balance with $0.01 \mathrm{~g}$ resolution in indication. Buoyancy corrections for dry nitrogen were applied.

The PS was placed in solvent the afternoon before the light scattering measurements were made. The solution bottles were quiescent over night, then stirred with magnetic stirrers for about 30 min the next morning. The partial specific volume for PS in toluene tabulated as $0.917 \mathrm{~mL} / \mathrm{g}$ by Brandrup and Immergut [8], and toluene density versus temperature tabulated from several sources by Riddick and Bunger [9], were applied to compute solution concentrations at $25.0^{\circ} \mathrm{C}$.

Solvent and solution samples were filtered into light scattering cells. The solvent and solution samples were filtered through filtering assemblies with double thicknesses of Millipore Fluoropore membrane (Millipore Corp., Bedford, MA) with $0.22 \mu \mathrm{m}$ average pore size.

\subsubsection{Determination of $d n / d c$}

The differential refractive index for SRM $706 a$ in toluene at $25^{\circ} \mathrm{C}$ for light of $632.8 \mathrm{~nm}$ wavelength was determined using a LDC/Milton Roy Chromatix KMX-16 (Thermo-Separation Products, Sunnyvale, CA) differential refractometer. The differential refractometer was calibrated against aqueous $\mathrm{NaCl}$ solutions.

Refractive increments versus concentration for several aqueous electrolyte solutions at several wavelengths of light were reported by Kruis [10]. Refractive increments for the same solutions at the He-Ne laser wavelength, $\lambda=632.8 \mathrm{~nm}$, have been determined from interpolation of the data in the Kruis tables [11,12]. A cubic equation for these refractive increments as a function of $\mathrm{NaCl}$ concentration in aqueous solution at $25^{\circ} \mathrm{C}$ is given in the instrument manual, and was used to compute the refractive increments of the aqueous $\mathrm{NaCl}$ solutions prepared as standards in calibrating the differential refractometer.

Mallinckrodt analytical reagent $\mathrm{NaCl}$ was dried in a vacuum oven at $90^{\circ} \mathrm{C}$ for three days in preparation to be used as a calibrant. The dried $\mathrm{NaCl}$ was then maintained in a vacuum desiccator except while taking salt samples to prepare solutions. Distilled water was degassed by boiling and left to cool to ambient temperature overnight in storage bottles tightly capped with zero headspace. The 
storage bottles had been leached out with several changes of boiling distilled water before being used to contain the degassed distilled water. Both salt and water components of each solution were measured gravimetrically, and atmospheric buoyancy corrections were applied to compute the concentrations as $\mathrm{g} \mathrm{NaCl} / 100 \mathrm{~g} \mathrm{H}_{2} \mathrm{O}$. Measurements in the differential refractometer were conducted on seven solutions ranging in concentration from $0.5 \mathrm{~g} \mathrm{NaCl} / 100 \mathrm{~g} \mathrm{H}_{2} \mathrm{O}$ to $2.0 \mathrm{~g} \mathrm{NaCl} / 100 \mathrm{~g} \mathrm{H}_{2} \mathrm{O}$ in intervals of $0.25 \mathrm{~g} \mathrm{NaCl} / 100 \mathrm{~g} \mathrm{H}_{2} \mathrm{O}$. The calculated refractive increments of the solutions were fitted to their average image displacements to generate a linear calibration equation of refractive increment versus image displacement, $d n / d x$.

Refractive increments between solvent and solutions of PS in toluene were determined on solutions which had been prepared on the morning of each day during which the measurements were conducted. The solutions were prepared by the procedure described in Section 4.1.1.

Image displacement measurements were conducted on six toluene solutions of SRM 706a varying in concentration from approximately $1 \mathrm{~g} / \mathrm{L}$ to $3 \mathrm{~g} / \mathrm{L}$ at $25^{\circ} \mathrm{C}$. An average was taken from eight individual image displacement measurements for each solvent versus solvent and solution versus solvent. The average image displacement determined for each solution was bracketed by the determination of average solvent versus solvent image displacements before and after that of the solution. The incremental image displacement by each solution was obtained by subtracting the mean of the bracketing solvent average image displacements from the average image displacement by the solution. The refractive increment of each solution was computed by application of the calibration equation to the incremental image displacement of the solution. Linear regression analysis of the refractive increments versus the concentrations of the solutions yielded a differential refractive index, $\mathrm{dn} / \mathrm{dc}=0.1089$ $\mathrm{mL} / \mathrm{g}$, for SRM 706a in toluene with a standard deviation of the mean of $0.0009 \mathrm{~mL} \mathrm{~g}$.

The differential refractive index at $632.8 \mathrm{~nm}$ for other comparable polystyrenes in toluene had also been determined earlier by others with values ranging from 0.106 $\mathrm{mL} / \mathrm{g}$ to $0.111 \mathrm{~mL} / \mathrm{g}[8]$. Our value is within the range of these reported values.

\subsubsection{Refractive Indices of Solvent and Calibrant}

The refractive indices of toluene and of benzene, the latter used as the calibrating standard, were derived from tabulated values for the $\mathrm{He}-\mathrm{Ne}$ laser wavelength, $632.8 \mathrm{~nm}$, at $23^{\circ} \mathrm{C}$ by Kaye and McDaniel [13]. Values for the temperature dependence of the refractive indices of toluene and of benzene at $633 \mathrm{~nm}$ were estimated from temperature dependence of the refractive indices of these two solvents at $589 \mathrm{~nm}$ tabulated by Riddick and Bunger [9]. The resulting calculated values for $\mathrm{dn} / \mathrm{dT}$ were in close agreement with those tabulated by Johnson and Smith for other spectral wavelengths [14]. These dn/dT coefficients were applied to the 
refractive indices tabulated at $23^{\circ} \mathrm{C}$ by Kaye and McDaniel to obtain the refractive indices at $25^{\circ} \mathrm{C}$. The resulting refractive indices calculated at $25^{\circ} \mathrm{C}$ and $632.8 \mathrm{~nm}$ wavelength were 1.494 for toluene, and 1.497 for benzene.

\subsubsection{Light Scattering Methods}

Light scattering measurements on the PS solutions in toluene were made on a Brookhaven Instrument Model BI-200 (Brookhaven Instrument Corp., Ronkonkoma, NY) light scattering apparatus with a $10 \mathrm{mw} \mathrm{He}-\mathrm{Ne}$ laser light source. Since the laser beam is vertically polarized, and a vertical polarizer is used in the detector optics, we have $V_{v}$ polarization for the scattered light intensity.

The temperature was controlled at $25.0^{\circ} \mathrm{C}$ in all experiments with PS in toluene. In all experiments, the intensity measuring system was calibrated with the intensity of the light scattered from the beam at $90^{\circ}$ angle by a benzene standard cell, and the scattering intensity from each solvent and solution sample was measured at ten angles in the range from $30.0^{\circ}$ to $142.5^{\circ}$.

\subsection{Analysis of Light Scattering Data}

Light scattering data at $V_{v}$ polarization from polymer solutions of concentration $c$ and scattering angle $\Theta$ may be analyzed by fitting the scattering signal $I(\Theta, c)$ to [15]

$$
I(\Theta, c)=I(\Theta, 0)+c I_{G} /\left\{(\sin \Theta) \sum C_{i j}{ }^{i} \sin ^{2 i j}(\Theta / 2)\right\} \text {. }
$$

In eq. (1), $I_{G}$ is the scattering signal from the benzene working standard at $\Theta=90^{\circ}$.

We must first decide how many terms on the right-hand side must be included to provide an adequate fit to the experimental data. The dependence of $c / I_{c}$, where $I_{c}=$ $\sin \Theta[I(\Theta, c)-I(\Theta, 0)] / I_{G}$, upon $c$ and upon $\sin ^{2}(\Theta / 2)$ reflects solute-solvent interactions and solute size, respectively. Accordingly, preliminary scattering data for SRM 706a were first analyzed as $c / l_{c}$ versus $\sin ^{2}(\Theta / 2)$ at constant concentration and versus $c$ at constant scattering angle, to see whether a linear expansion (i.e., retaining only $C_{00}$, $\mathrm{C}_{01}$, and $\mathrm{C}_{10}$ ) would provide an adequate fit. The analysis revealed that the linear approximation was adequate at concentrations below $1.6 \mathrm{~g} / \mathrm{L}$ for SRM 706a.

Thus we used for the final analysis

$$
I(\Theta, c)=I(\Theta, 0)+c I_{G} /\left\{\sin \Theta\left(C_{00}+C_{01} \sin ^{2}(\Theta / 2)+C_{10} c+C_{11} c \sin ^{2}(\Theta / 2)\right\}\right.
$$

The coefficients in eq. (2) are related to the $M_{w}$, mean-square radius of gyration 
of the polymer, $R_{G}{ }^{2}$, and the second virial coefficients, $A_{2}$, by [15-18]:

$$
\begin{aligned}
& M_{w}=\left(K C_{00}\right)^{-1} \\
& R_{G}{ }^{2}=3\left[\lambda_{0} /(4 \pi n)\right]^{2} C_{01} / C_{00} \\
& A_{2}=1 / 2 K C_{10} \\
& K=4 \pi^{2} n_{B}{ }^{2}(d n / d c)^{2} /\left(\lambda_{0}{ }^{4} N_{A} V_{v}^{B}\right)
\end{aligned}
$$

where:

$\lambda_{0}$ is the wavelength in vacuum of the scattered light, $632.8 \mathrm{~nm}$ in this work, $n$ and $n_{B}$ are the indices of refraction of the solvent and benzene taken as 1.494 and 1.497, respectively calculated as described in $4.1 .3, \mathrm{dn} / \mathrm{dc}$ is the differential refractive index of the solution, measured as described in 4.1.2, $\mathrm{N}_{\mathrm{A}}$ is Avogadro's number, taken as 6.022 $\times 10^{23} / \mathrm{mol}, V_{v}{ }^{B}$ is the Rayleigh ratio for the vertically polarized scattering of vertically polarized light from benzene, used for calibration and obtained as described in the following paragraph.

The "vertical-vertical" Rayleigh ratio $V_{v}$ is related to the Rayleigh ratio $R_{v}$ for the unpolarized scattering of vertically polarized $\mathrm{He}-\mathrm{Ne}$ laser and the depolarization ratio $\rho_{v}$ for polarized light by:

$$
V_{v}^{B}=R_{v}^{B} /\left(1+\rho_{v}\right)
$$

Using the published [13] values for benzene

$$
R_{v}^{B}=12.6_{3} \times 10^{-6} \mathrm{~cm}^{-1}
$$

and $\rho_{v}=0.265$, we obtain

$$
V_{v}^{B}=9.98 \times 10^{-6} \mathrm{~cm}^{-1}
$$

\subsection{Results for SRM 706a and SRM 706}

Four sets of light scattering solutions were made from SRM 706a using toluene as solvent. Each set consisted of five independently made up solutions. The light scattering on each solution set was run twice, usually on consecutive days. The polymer for each solution within each set was taken from the same sample vial but for each set a different sample vial was used. Intensities were measured at ten scattering 
angles in the range from $30.0^{\circ}$ to $142.5^{\circ}$. The scattered intensities in each light scattering run were fitted by least squares to eq. (2), and the results were used to calculate $M_{w}, A_{2}$ and $R_{g}$ using eq. (3)-(7). The values of $M_{w}, A_{2}$, and $R_{g}$ obtained from the eight runs were then averaged. The resulting mean values and sample standard deviations are given in Table 1. Light scattering gave a $M_{w}$ of $2.85 \times 10^{5} \mathrm{~g} / \mathrm{mol}$ with a sample standard deviation of $0.054 \times 10^{5} \mathrm{~g} / \mathrm{mol}$, a value of $A_{2}$ of $0.000411 \mathrm{~mol} \mathrm{~mL} / \mathrm{g}^{2}$ with a sample standard deviation of $0.000025 \mathrm{~mol} \mathrm{~mL} / \mathrm{g}^{2}$ and a value of $R_{\mathrm{g}}$ of $27.8 \mathrm{~nm}$ with a sample standard deviation of $0.95 \mathrm{~nm}$. The value of $A_{2}$ is in good agreement with that given by Fetters et al [16 ] in their review of thermodynamic properties of polymer solutions . Fetters et al give $A_{2}=0.000399 \mathrm{~mol} \mathrm{~mL} / \mathrm{g}^{2}$. They also quote a value of $R_{g}$ of $21.17 \mathrm{~nm}$. Our value of $R_{g}$ is higher than this. Their value of $R_{g}$ is for a narrow MWD polymer. SRM 706a is a broad MWD polymer and thus the higher molecular weight polymers dominate the values of $R_{\mathrm{g}}$.

The standard deviation of the mean for the average $M_{w}$ is $0.019 \times 10^{5} \mathrm{~g} / \mathrm{mol}$. In compliance with the NIST policy [17] on reporting uncertainties in measurement, the standard uncertainty due to variance among the four $\mathrm{M}_{\mathrm{w}}$ determinations is computed as the standard deviation of the mean. This standard uncertainty is multiplied by a coverage factor of 2 to obtain the component of expanded uncertainty listed in Table 3.

Two of the remaining bottles of SRM 706 were chosen for light scattering measurements. Independent sets of light scattering solutions were prepared from SRM 706 in toluene. Measurements were made in the same way as described for SRM 706a above. The resulting mean values and sample standard deviations are also given in Table 2. Light scattering gave $M_{w}$ of $2.87 \times 10^{5} \mathrm{~g} / \mathrm{mol}$ with a sample standard deviation of $0.068 \times 10^{5} \mathrm{~g} / \mathrm{mol}$ and a value of $A_{2}$ of $0.000421 \mathrm{~mol} \mathrm{~mL} / \mathrm{g}^{2}$ with a standard deviation of $0.000039 \mathrm{~mol} \mathrm{~mL} / \mathrm{g}^{2}$. The standard deviation of the mean of $M_{w}$ for the average SRM 706 is $0.034 \times 10^{5} \mathrm{~g} / \mathrm{mol}$.

Thus, SRM 706a and SRM 706 have indistinguishable $M_{w}$ 's.

In the original certificate and report on SRM 706 from 1966 [18], Mclntyre found $M_{w}$ as measured by light scattering to be $2.58 \times 10^{5} \mathrm{~g} / \mathrm{mol}$ and $M_{w}$ measured by sedimentation was $2.88 \times 10^{5} \mathrm{~g} / \mathrm{mol}$. The 1997 value of $M_{w}$ from light scattering is about $0.29 \times 10^{5} \mathrm{~g} / \mathrm{mol}$ greater than the $M_{w}$ obtained by light scattering in 1967 but nearly identical to the $M_{w}$ obtained by sedimentation in 1967. Mcintyre gave no detailed description of his centrifugation or light scattering in his 1967 paper [1]. Thus, we can not comment on the differences within his work and the difference between his light scattering results and ours.

\subsection{Estimation of Uncertainties Due to Systematic Effects in the Light Scattering}

We list the likeliest sources of systematic uncertainty in the determination of the 
$M_{w}$ of SRM 706a by light scattering described in the preceding sections. We attempt to estimate upper limits for their magnitudes. For this purpose, we employ a scheme similar to that used in Ref. [15] for the estimation of systematic uncertainties in SRM's 1482,1483 and 1484. These uncertainties are listed in Table 3 for SRM 706a.

\subsubsection{Indices of Refraction}

Following Ref. [15], we estimate that $0.1 \%$ is a proper upper limit for systematic uncertainties in $\mathrm{M}_{\mathrm{w}}$ arising from uncertainties in the literature values of solvent index of refraction.

\subsubsection{Literature Value of $\mathrm{dn} / \mathrm{dc}$ for Aqueous $\mathrm{NaCl}$ and Calibration of the Differential Refractometer}

Calibration of the differential refractometer required interpolation of the data of Ref. [10] to the $632.8 \mathrm{~nm}$ wavelength used for the light-scattering measurements. We estimate the uncertainty in the interpolated values of $\mathrm{dn} / \mathrm{dc}$ as $0.6 \%$, due primarily to uncertainties in the interpolation process. The calibration factor determined for our differential refractometer had a relative standard deviation (rsd) of $0.097 \%$. Combining the above uncertainties with an allowance for possible linear uncertainties in the refractometer, we estimate that a proper upper limit for uncertainty to $\mathrm{dn} / \mathrm{dc}$ from this contribution is $1 \%$. Considered as a 95 percent confidence interval estimate, this quoted uncertainty provides an expanded uncertainty in $\mathrm{dn} / \mathrm{dc}$ of $1 \%$ [17] which would contribute $2 \%$ or $0.06 \times 10^{5} \mathrm{~g} / \mathrm{mol}$ expanded uncertainty in $\mathrm{M}_{\mathrm{w}}$ determination.

\subsubsection{Measured Value of $d n / d c$ of SRM 706a}

The differential refractive index dn/dc of SRM 706a in toluene at a temperature of $25^{\circ} \mathrm{C}$. was determined as described in 4.1.2. The mean value obtained for $\mathrm{dn} / \mathrm{dc}$ was $0.1089 \mathrm{~mL} / \mathrm{g}$, with a standard deviation of the mean of $0.00092 \mathrm{~mL} / \mathrm{g}$ or $0.85 \%$. As the $\mathrm{dn} / \mathrm{dc}$ appears raised to the second power in the $\mathrm{M}_{\mathrm{w}}$ calculation, the uncertainty in the calculated $M_{w}$ resulting from the standard deviation in the mean of $d n / d c$ is estimated as twice the estimated standard uncertainty in the $\mathrm{dn} / \mathrm{dc}$, or $1.7 \%$. The expanded uncertainty from this measurement is $3.4 \%$ or $0.10 \times 10^{5} \mathrm{~g} / \mathrm{mole}$.

\subsubsection{Wavelength of Radiation}

For the He-Ne laser employed in this work, uncertainties in the wavelength of the radiation are completely negligible compared with uncertainties from other sources.

\subsubsection{Rayleigh Ratio of Benzene}

For benzene at $632.8 \mathrm{~nm}$, Ref. [13] gives: $R_{v, v+H}=12.6_{3} \times 10^{-6} \mathrm{~cm}^{-1}$ and $\rho_{v}=$ 
0.265 , giving $R_{V, v}=R_{V, V+H} /\left(1+\rho_{v}\right)=9.98 \times 10^{-6} \mathrm{~cm}^{-1}$. From here on we abbreviate $R_{V, V+H}$ and $\rho_{v}$ by $R$ and $\rho$, respectively. The authors of Ref. [13] report that their $R$-values are accurate to $2 \%$ (systematic). They quote a relative standard uncertainty for their Rvalue for benzene of $0.21 / 12.63$, or $1.7 \%$. They do not give estimates of either accuracy or precision for their values of $\rho$. However, $\rho$ is obtained as the ratio of two intensities, the larger of which is, or is close to, the intensity measured for the determination of $R$. The photomultiplier detectors were apparently operated in the current mode, and it seems reasonable to suppose that the absolute uncertainty in the smaller intensity is the same as that of the larger, and that the relative uncertainty in the larger is the same as that in $R$. Then if $r$ is the relative standard deviation (rsd) of $R$, we have for the standard deviation in $\rho: \operatorname{sd}(\rho)=r V\left(1+\rho^{2}\right)$ and $r s d(1+\rho)=[r /(1+\rho)] V\left(1+\rho^{2}\right)$, and combining this with the rsd in $R$, we have $\operatorname{rsd}\left(R_{v, v}\right)=[r /(1+\rho)] / 2\left(1+\rho+\rho^{2}\right)$, which is about $1.3 r$ for $p=0.265$. The product, $1.3 r$ $=1.3 \times 1.7 \%$ yields a standard uncertainty $2.2 \%$. This standard uncertainty combined (by root-sum-squares) with the stated standard uncertainty of $2 \%$ for the R-values [13] yields a standard uncertainty of $3 \%$ or $0.09 \times 10^{5} \mathrm{~g} / \mathrm{mol}$. Applying the coverage factor of 2 to this standard uncertainty we obtain a expanded uncertainty of $0.18 \times 10^{5} \mathrm{~g} / \mathrm{mol}$.

\subsubsection{Polarizer Uncertainties}

There are four of these: First, the "vertically polarized" laser beam actually contains "horizontally polarized" components for two reasons: First, the polarizer inside the laser head lets through a small fraction $\epsilon$ of the "wrong" polarization; Second, the principal axis of polarization of the light from the laser may not be exactly perpendicular to the plane of the incident and scattered beams. Both will cause light assumed to be vertically polarized to contain a small admixture of horizontally polarized light. The effect upon scattering signals from SRM 706a will be slight, but the effect upon the benzene calibration signals is to change the effective Rayleigh ratio that should have been used from the $R_{W}$ value toward the $R_{V, v+H}$ value. The resulting uncertainty in $M_{w}$ is $\rho \in /(1-\varepsilon)$ for the first effect and $\operatorname{ptan}^{2} \alpha$, where $\alpha$ is the angular missetting, for the second. The uncertainty from both effects together is $p[\epsilon /(1-\epsilon)+$ $\left.\tan ^{2} \alpha\right]$.

Second, in an exactly analogous way, the analyzing polarizer in front of the detector may be nonideal and/or mispositioned. In this case, let $\delta$ be the contribution from the nonideality of the polarizer, and let $\beta$ be the angle of missetting. The resulting expression for the uncertainty is then: $\rho\left[\delta /(1-\delta)+\tan ^{2} \beta\right]$.

Finally, since all these uncertainties are of the same sign, we add them to get: $\rho\left[\epsilon /(1-\epsilon)+\tan ^{2} \alpha+\delta /(1-\delta)+\tan ^{2} \beta\right]$. Take $\epsilon=\delta=1 / 500, \alpha=5^{\circ}$, and $\beta=3^{\circ}$, and $\rho=0.265$. Then the uncertainty is $0.265[.0020+.0077+.0020+.0027]=0.0038=0.4$ $\%$ with at least a $95 \%$ level of confidence considering the liberal boundaries assigned to the constituent uncertainties and their combination by linear summation instead of 
root-sum-of-squares. This quoted uncertainty provides an estimated expanded uncertainty of $0.4 \%$ or $0.01 \times 10^{5} \mathrm{~g} / \mathrm{mol}$.

\subsubsection{Ratio of Working Standard Scattering to Sample Scattering}

Since photon counting techniques were employed, there should be no systematic uncertainties from this source. Random uncertainties are reflected in the overall random uncertainty of the $M_{w}$.

\subsubsection{Solvent Density}

For the density of toluene at $25^{\circ} \mathrm{C}$, we used the value $0.86231 \mathrm{~g} / \mathrm{mL}$ as given in reference [9]. We estimate that this value is accurate to $0.1 \%$, or better. The resulting expanded uncertainty on $M_{w}$ is just $0.1 \%$ or less than $0.01 \times 10^{5} \mathrm{~g} / \mathrm{mol}$.

\subsubsection{Solute Weights}

For the runs actually used in the final determination of $M_{w}$ for SRM 706a, the concentrations employed were nominal 0.5 to $2.0 \mathrm{~g} / \mathrm{L}$ for SRM $706 \mathrm{a}$. Solvent weights were chosen so that the solute weights were always about $0.05 \mathrm{~g}$. Using the uncertainty limit of $0.1 \mathrm{mg}$ we usually assign to the balance used to weigh the PS samples, we have uncertainties in the solute weights of $0.2 \%$ (and negligible uncertainties in solvent weights). The resulting expanded uncertainty in $M_{w}$ is about $0.2 \%$ or less than $0.01 \times 10^{5} \mathrm{~g} / \mathrm{mol}$.

\subsubsection{Reflection Correction}

The refractive index of toluene at $23^{\circ} \mathrm{C}$ and $632.8 \mathrm{~nm}$ wavelength is given [13] as 1.4940. The refractive index of the sample cell is given by the vendor as 1.474 at the $589 \mathrm{~nm}$ wavelength. Although the temperature of the toluene, and the wavelength for the refractive index of the cell, in this case are not correct for our experiments, these values should be adequate to estimate what will turn out to be an extremely small uncertainty. Substitution of these two refractive indices into Fresnel's equation for reflection from an interface between two transparent dielectrics [19] yields a reflectance factor $f=2 \times 10^{-4}$. Comparison calculations of $M_{w}$ with and without this correction for SRM 706a show the resulting uncertainty to be less than $0.01 \%$.

\subsubsection{Instrumental Misalignment}

In section 4.1.4 we discussed problems with instrument alignment in this system. For the geometry of the Brookhaven light scattering instrument, it is expected that any deviation from constancy of $I \sin (\Theta)$ is indicative of instrument misalignment. Our $\mid \sin (\Theta)$ measurements show a maximum uncertainty of $1.5 \%$ in the intensity compared 
to the intensity at 90 degrees as a function of angle. The maximum misalignment we estimate from this to be 0.5 degree. We have looked at two sets of data and they both show that for a systematic angle variation of +0.5 degrees the $M_{w}$ varies by $2.1 \%$ and that for a systematic angle variation of -0.5 degrees the $M_{w}$ varies by $-2.1 \%$. Since the error seems more random, we expect the correction to be less. Thus, we expect an expanded uncertainty arising from instrument misalignment to be less than $2.1 \%$ or $0.06 \times 10^{5} \mathrm{~g} / \mathrm{mol}$ for SRM 706a.

\subsubsection{Refraction Correction}

A detailed analysis of the optical geometry of the light scattering instrument employed in this work can not be carried out, since the main detector optics unit was inaccessible. However, rough analyses based on assumptions about the internal geometry of the detector unit lead to an uncertainty of about $0.3 \%$. A reasonable uncertainty limit might then be about twice this, or $0.6 \%$, from which we estimate an expanded uncertainty of $0.6 \%$ or $0.02 \times 10^{5} \mathrm{~g} / \mathrm{mol}$ on $M_{w}$ due to refraction uncertainty.

\subsubsection{Anisotropy of Solute}

We know of no reported optical anisotropy of polystryenes in toluene.

\subsubsection{Cutoff of Virial Expansion for SRM 706a}

As described in section 4.2., the solution concentrations used for the final analyses were limited to a region where linear terms in $c$ and $X \equiv \sin ^{2}(\Theta / 2)$ appeared to suffice. To check this further, we used our data on $A_{2}$ to estimate values of $A_{3}$.

Yamakawa [20] suggests $A_{3}$, the third virial may be represented in the Flory form as $A_{3}$ $=k_{f}\left(A_{2}\right)^{2} M$ where $k_{f}$ is between 0.5 and 1.0. We take $k_{f}$ to be 0.75 . At the highest concentration we used for the light scattering measurements, the contribution from $A_{3}$ was then $7 \%$ of the scattering difference between solution and solvent and at the lowest concentration it was $0.6 \%$ of the scattering difference.

We refit the data on 6 of our runs dropping off the highest concentration and found a change in the $\mathrm{M}_{\mathrm{w}}$ of $+0.3 \%,-0.3 \%,+1 \%,-.6 \%,-.3 \%$, and $0 \%$. We take the largest uncertainty in the $\mathrm{M}_{\mathrm{w}}$ arising from the cutoff of the virial expansion to be less than $1.0 \%$ or $0.03 \times 10^{5} \mathrm{~g} / \mathrm{mol}$ and take that to be the expanded uncertainty.

\subsubsection{Solute Degradation}

By their nature light scattering experiments are of short duration. A number of solutions can be prepared and run by light scattering in a single day. Solutions were made up one day and often run the next day. The second run on the same solutions often occurred the following day or a few days later. No effect was seen of allowing the 
solutions to sit around for many days. Furthermore, in doing the SEC studies on PS solutions, we see no indication of degradation of the polymer over periods of weeks.

As long as we prepare fresh solutions and run them within a day or two, we expect no problem. This was our practice during the entire series of light scattering experiments. We attribute no error arising from degradation.

\subsubsection{Summary}

The standard deviation of the mean of the determined $\mathrm{M}_{\mathrm{w}}$ values, from analysis of variance of the experimental data, and the systematic uncertainties obtained from section 4.4.1 through 4.4.15 are listed in Table 3 for SRM 706a.

The combined expanded uncertainties of SRM 706a are computed as root-sumof-squares of the component expanded uncertainties following the formal NIST policy for evaluating and expressing uncertainty in measurements [17]. We find that the combined expanded uncertainty of SRM $706 a$ is $0.23 \times 10^{5} \mathrm{~g} / \mathrm{mol}$.

\subsection{Conclusions}

The $M_{w}$ of SRM 706a, a polystyrene, was determined to be $2.85 \times 10^{5} \mathrm{~g} / \mathrm{mol}$ by light scattering, with a combined expanded uncertainty $0.23 \times 10^{5} \mathrm{~g} / \mathrm{mol}$. This is in agreement with the $M_{w}$ measured on SRM 706 in 1966 by ultracentrifugation and with a value of $M_{w}$ on SRM 706 obtained by light scattering recently. From the SEC study, we also conclude there is no difference between SRM 706 and SRM 706a and that the vials containing SRM 706a are indistinguishable from each other.

\subsection{References}

[1] D. Mclntyre, Jour. Research of NBS,71A,43 (1967)

[2] M. G. Natrella, "Experimental Statistics", National Bureau of Standards Handbook 91, U.S. Department of Commerce, 1963

[3] L. Huber, "Application of the Diode-Array Detector in HPLC", Hewlett Packard, France, 08/89, pg. 89-100

[4] Barry J. Bauer, Brian Dickens and William Blair, "Chromatographic Examination of Intaglio Inks, Resins and Varnishes", NISTIR 4949, 1991

[5] "PC-OMNITAB: An Interactive System for Statistical and Numerical Data Analysis," available from National Technical Information Service, Technology Administration, U.S. 
Department of Commerce, Springfield, VA 22161

[6] "DATAPLOT: Interactive Graphics \& Data Analysis Program, "available on the web from http://uww.nist.gov/it//div898/software/dataplot or from National Technical Information Service, Technology Administration, U.S. Department of Commerce, Springfield, VA 22161

[7] Charles M. Guttman, William R. Blair, John R. Maurey, "Recertification of SRM 1482a, a Polyethylene", NISTIR 6054, 1997, available from National Technical Information Service, Technology Administration, U.S. Department of Commerce, Springfield, VA 22161

[8] J. Brandrup and E. H. Immergut, editors, "Polymer Handbook, Third Edition", John Wiley and Sons, New York, 1989, section Vill pg 93

[9] J. A. Riddick and W. B. Bunger, "Organic Solvents, Phys. Properties and Methods of Purification," Wiley-Interscience, New York, 1970, Table 58, pg 145. or Table 30, pg 107.

[10] A. Kruis, Z. Physik. Chem. B 34, 13-50 (1936).

[11] R. J. Anderson, Appl. Opt. 8 , 1508-1509 (1969).

[12] LDC Analytical, "Instruction Manual for KMX-16 Laser Differential Refractometer," Section 5.3, "Calculation of Calibration Constant," Riviera Beach, FL, USA.

[13] W. Kaye and J. B. McDaniel, Appl. Opt., 13, 1934-1937 (1974).

[14] B. L. Johnson and J. Smith, "Refractive Indices and Densities of Some Common Polymer Solvents," Chap. 2 in "Light Scattering from Polymer Solutions," Ed M. B. Huglin, Academic Press, London and N.Y., (1972).

[15] Charles C. Han, Herman L. Wagner, and Peter H. Verdier, "The Characterization of Linear Polyethylene SRM's 1482, 1483, and 1484 III Weight-Average Molecular Weights by Light Scattering." NBS Special Publication 260-61, P.H. Verdier and H.L. Wagner ed. December 1978 or National Bureau of Standards, Journal of Research, Vol. 83, No. 2, March - April, 1978, pg. 5-193.

[16] Fetters et al, "Molecular Weight Dependence of Hydrodynamic and Thermodynamic Properties for Well-Defined Linear Polymers in Solution" J Phy Chem Ref Data, Vol 23 (4) July -Aug 1994 pg 619-640. 
[17] B. N. Taylor and C. E. Kuyatt, "Guidelines for Evaluating and Expressing the Uncertainty of NIST Measurement Results." NIST Technical Note 1297, January 1993, available from National Technical Information Service, Technology Administration, U.S. Department of Commerce, Springfield, VA 22161

[18] National Bureau of Standards Certificate Standard Reference Material 706, Polystyrene (Broad Molecular Weight Distribution), 1979 (Editorial revision of previous certificate).

[19] Brookhaven Instrument Corp., "Instruction Manual for Laser Light Scattering Goniometers," May 1984, Third Printing.

[20] H. Yamakawa, "Modern Theory of Polymer Solutions," Harper \& Row, N.Y., (1971). 
Table 1

$M_{w}, A_{2}$, and $R_{g}$ for SRM 706a

\begin{tabular}{|c|c|c|c|}
\hline $\begin{array}{l}\text { Run } \\
\text { Label }\end{array}$ & $\begin{array}{l}\mathrm{M}_{\mathrm{w}} \times 10^{-5} \\
\mathrm{~g} / \mathrm{mol}\end{array}$ & $\begin{array}{c}\mathrm{A}_{2} \\
\mathrm{~mL} \mathrm{~mol} / \mathrm{g}^{2}\end{array}$ & $\underset{\mathrm{nm}}{\mathrm{R}_{\mathrm{g}}}$ \\
\hline L951213 & 2.85 & 0.000437 & 26.9 \\
\hline L951214 & 2.91 & 0.000436 & 28.7 \\
\hline L960130 & 2.76 & 0.000379 & 27.4 \\
\hline L960131 & 2.77 & 0.000371 & 27.9 \\
\hline L960209 & 2.86 & 0.000404 & 29.2 \\
\hline L950212 & 2.87 & 0.000420 & 28.3 \\
\hline L960220 & 2.87 & 0.000420 & 26.3 \\
\hline L960221 & 2.89 & 0.000422 & 27.9 \\
\hline Average & 2.85 & 0.00411 & 27.8 \\
\hline $\begin{array}{l}\text { Sample } \\
\text { Standard }\end{array}$ & & & \\
\hline Deviation & 0.054 & 0.000025 & 0.95 \\
\hline $\begin{array}{l}\text { Standard } \\
\text { Deviation } \\
\text { of Mean }\end{array}$ & 0.019 & & \\
\hline
\end{tabular}


Table 2

$M_{w}, A_{2}$, and $R_{g}$ for SRM 706

$\begin{array}{lccc}\begin{array}{l}\text { Run } \\ \text { Label }\end{array} & \begin{array}{c}\mathrm{M}_{\mathrm{w}} \times 10^{-5} \\ \mathrm{~g} / \mathrm{mol}\end{array} & \begin{array}{c}\mathrm{A}_{2} \\ \mathrm{~mL} \mathrm{~mol} / \mathrm{g}^{2}\end{array} & \begin{array}{c}\mathrm{R}_{\mathrm{g}} \\ \mathrm{nm}\end{array} \\ \text { L951102 } & 2.94 & 0.000457 & 27.5 \\ \text { L951103 }^{\mathrm{a}} & 2.91 & 0.000445 & 26.5 \\ \text { L951121 }^{2} & 2.84 & 0.000409 & 27.9 \\ \text { L951122 }^{\mathrm{a}} & 2.79 & 0.000371 & 25.1\end{array}$

$\begin{array}{llll}\text { Average } & 2.87 & 0.000421 & 26.75\end{array}$

Sample

Standard

Deviation

$0.068 \quad 0.000039$

1.25 
Table 3

Contributions to Combined Expanded Uncertainty

of the $M_{w}$ of SRM 706a

Source of uncertainty

Expanded Uncertainty from:

Standard deviation of the mean $\mathrm{M}_{\mathrm{w}}$

Solvent index of refraction

Calibration of differential refractometer

Differential refractive index

Wavelength of light

Rayleigh ratio of scattering standard

Light polarizers

Ratio of standard scattering

to sample scattering

Solvent density

Solute weights and solvent weights

Light reflection

Optical alignment

Refraction correction

Anisotropy of polymer in solution

Truncation of virial expansion

Solute degradation
Contribution

$10^{-5} \mathrm{~g} / \mathrm{mol}$

0.04

$<0.01$

0.06

0.10

$<0.01$

0.18

0.01

$<0.01$

$<0.01$

$<0.01$

$<0.01$

0.06

0.02

$<0.01$

0.03

$<0.01$

Combined expanded uncertainty of $M_{w}$ of $0.23 \times 10^{5} \mathrm{~g} / \mathrm{mol}$ by root-sum-of-squares [17]. 
This Standard Reference Material (SRM) is intended primarily for use in calibration and performance evaluation of instruments used to determine the molar mass and molar mass distribution. A unit of SRM 706a consists of approximately $18 \mathrm{~g}$ of polystyrene pellets. Each pellet weighs approximately $80 \mathrm{mg}$.

Certified Value: The certified value for $M_{w}$ is based on original results from light scattering and ultracentrifugation measurements on SRM 706 [1,2] and a remeasurement of 706a using light scattering [3]. The certified value represents the highest level of confidence NIST has in its accuracy in that all known or suspected sources of bias have been fully investigated or accounted for.

The certified measurement uncertainty is expressed as a combined expanded uncertainty with a coverage factor $k=2$, calculated in accordance with NIST procedure [4]. Type A and Type B contributions to the expanded uncertainty of the certified molar mass include the uncertainties in the light scattering method due to the Rayleigh ratio of the scattering standard, optical alignment, and calibration of the differential refractometer.

Table 1. Certified Value

$\begin{array}{cc}\text { Property* } & \text { Certified Value and Uncertainty } \\ \text { mass-average molar mass }\left(\mathrm{M}_{w}\right), & 2.85 \times 10^{5} \mathrm{~g} / \mathrm{mol}=0.23 \times 10^{5} \mathrm{~g} / \mathrm{mol}\end{array}$

*Expressed as molar mass, previously expressed as molecular weight [5].

Expiration of Certification: The certification of SRM 706a is valid, within the measurement uncertainties specified, until 26 August 2003 provided that the SRM is handled in accordance with the storage instructions given in this certificate. This certification is nullified if the SRM is modified or contaminated.

Maintenance of SRM Certification: NIST will monitor this SRM over the period of its certification. If substantive technical changes occur that affect the certification before expiration of this certificate, NIST will notify the purchaser. Return of the attached registration card will facilitate notification.

Technical coordination leading to certification of this SRM was provided by B.M. Fanconi of the NIST Polymers Division.

Technical measurement and data interpretation were provided by C.M. Gutman, W.R. Blair, and J.R Maurey of the NIST Polymers Division.

The support aspects involved in the preparation, certification, and issuance of this SRM were coordinated through the Standard Reference Materials Program by R.J. Gettings.

Gaithersburg, MD 20899

Certificate Issue Date: 2 September 1998
Thomas E. Gills, Chief Standard Reference Materials Program 
Reference Values: The reference values for intrinsic viscosity were determined by measurements made on SRM 706 in the solvent benzene at $25^{\circ} \mathrm{C}$ and in cyclohexane at $35^{\circ} \mathrm{C}$. Details of the measurements are given in references [1,2]. The reference values are the best estimate of the true value, however all known or suspected sources of bias have not been fully investigated. The uncertainties in the reference values for intrinsic viscosity are expressed as the standard deviation of the mean.

Table 2. Reference Values

Property

intrinsic viscosity [ $\eta$, in benzene at $25^{\circ} \mathrm{C}$

ntrinsic viscosity $[\eta]$, in cyclohexane at $35^{\circ} \mathrm{C}$
Reference Value and Standard Deviation of the Mean

$93.70 \mathrm{~mL} / \mathrm{g} \pm 0.19 \mathrm{~mL} / \mathrm{g}$

$39.50 \mathrm{~mL} / \mathrm{g} \pm 0.10 \mathrm{~mL} / \mathrm{g}$

Storage: The SRM should be stored in the original bottle with the lid tightly closed under normal laboratory conditions.

Homogeneity and Characterization: The homogeneity of SRM 706a was tested using size exclusion chromatography (SEC) analysis of solutions in tetrahydrofuran at $30^{\circ} \mathrm{C}$. The characterization of this polymer is described in reference [3]. SRM 706a is a reblending and bottling of the remaining stock of polystyrene used to produce SRM 706.

The SRM 706 polystyrene was originally prepared by thermal polymerization of styrene at $140{ }^{\circ} \mathrm{C}$ to $37 \%$ conversion. Ash content is less than $0.001 \%$. Volatile content is approximately $0.8 \%$. Determinations of molar mass and intrinsic viscosity are based on the mass of the polystyrene pellets uncorrected for volatiles.

\section{REFERENCES}

[1] National Bureau of Standards, SRM 706 Polystyrene (Broad Molecular Weight Distribution) Certificate, 1967.

[2] McIntyre, D., Jour. Research of NBS, 71A, No. 1, p. 43, (1967).

[3] Guttman, C.M., Blair, W.R, and Maurey, J.R., "Recertification of SRM 706a, A Polystyrene," NISTIR 6091.

[4] Guide to the Expression of Uncertainty in Measurement, ISBN 92-67-10188-9, Ist Ed. ISO, Geneva, Switzerland, (1993): see also Taylor, B.N., and Kuyatt, C.E., "Guidelines for Evaluating and Expressing the Uncertainty of NIST Measurement Results," NIST Technical Note 1297, U.S. Government Printing Office. Washington DC, (1994).

[5] Taylor, B.N., "Guide for the Use of the International System of Units (SI)," NIST Special Publication 811, 1995 Ed. (April 1995).

Users of this SRM should ensure that the certificate in their possession is current. This can be accomplished by contacting the SRM Program at: Telephone (301) 975-6776 (select "Certificates"), Far (301) 926-4751, e-mail srminfo@inist.gov,or via the Internet http://ts.nist.gov/srm. 

